\title{
ON CHRISTOFFEL FUNCTIONS AND RELATED QUANTITIES FOR COMPACTLY SUPPORTED MEASURES
}

\author{
D. S. LUBINSKY
}

\begin{abstract}
Let $\mu$ be a compactly supported positive measure on the real line, with associated orthogonal polynomials $\left\{p_{n}\right\}$. Without any global restrictions such as regularity, we discuss convergence in measure for

(i) ratio asymptotics for Christoffel functions;

(ii) the Nevai operators (aka the Nevai condition);

(iii) universality limits in the bulk.

We also establish convergence a.e. for sufficently sparse subsequences of Christoffel function ratios.
\end{abstract}

Orthogonal Polynomials on the real line, Christoffel functions, universality limits in the bulk. $42 \mathrm{C} 05$

\section{INTRODUCTION ${ }^{1}$}

Let $\mu$ be a positive measure on the real line, with compact support $\operatorname{supp}[\mu]$, and infinitely many points in its support. Then we may define orthonormal polynomials

$$
p_{n}(x)=\gamma_{n} x^{n}+\ldots, \gamma_{n}>0,
$$

satisfying

$$
\int p_{n} p_{m} d \mu=\delta_{m n}
$$

The measure $\mu$ is said to be regular in the sense of Stahl, Totik and Ullmann [29] if

$$
\lim _{n \rightarrow \infty} \gamma_{n}^{1 / n}=\frac{1}{\operatorname{cap}(\operatorname{supp}[\mu])},
$$

where $\operatorname{cap}(\operatorname{supp}[\mu])$ is the logarithmic capacity of the support of $\mu$. In particular, if the support is an interval $[a, b]$, the requirement is that

$$
\lim _{n \rightarrow \infty} \gamma_{n}^{1 / n}=\frac{4}{b-a} .
$$

For definitions of logarithmic capacity, and the associated potential theory, see [22], [23], [29].

At first this particular definition seems technical and obscure - to the extent that one might doubt the utility of the concept. There are numerous equivalent definitions of regularity, but (1.1) is used because it is relatively direct. An important monograph by Stahl and Totik [29] comprehensively explores regular measures and the asymptotics in of their orthogonal polynomials. More recent analysis appears in [24].

Regularity of a measure is a very weak global requirement. Thus the ErdösTurán criterion asserts that if $\mu^{\prime}>0$ a.e. in $\operatorname{supp}[\mu]$, then $\mu$ is regular. But far

Date: May 14, 2010.

${ }^{1}$ Research supported by NSF grant DMS0700427 and US-Israel BSF grant 2004353 
less guarantees regularity, and there are pure jump, and pure singularly continuous measures, that are regular.

Here is a very useful equivalent formulation of regularity: $\mu$ is regular, iff for every sequence of polynomials $\left\{P_{n}\right\}$, where $\operatorname{deg}\left(P_{n}\right) \leq n$, we have

$$
\limsup _{n \rightarrow \infty}\left[\left|P_{n}(x)\right| /\left(\int\left|P_{n}\right|^{2} d \mu\right)^{1 / 2}\right]^{1 / n} \leq 1,
$$

for quasi every $x \in \operatorname{supp}[\mu]$. Here quasi-every means except on a set of capacity 0 . When $\mathbb{C} \backslash \operatorname{supp}[d \mu]$ is regular for the Dirichlet problem, one can replace $\left|P_{n}(x)\right|$ by $\left\|P_{n}\right\|_{L_{\infty}(\operatorname{supp}[d \mu])}$. Thus, in an $n$th root sense, the sup norms of polynomials are comparable to their $L_{2}(d \mu)$ norms. Regularity of $\mu$ also permits asymptotics for $p_{n}(z)^{1 / n}$ outside $\operatorname{supp}[\mu]$, and on $\operatorname{supp}[\mu]$. In particular, regularity is equivalent to

$$
\limsup _{n \rightarrow \infty}\left|p_{n}(x)\right|^{1 / n}=1 \text { for quasi every } x \in \operatorname{supp}[\mu] \text {. }
$$

Perhaps most surprising of all, is the appearance of this concept in so many orthogonal polynomial asymptotics that have nothing to do with $n$th root asymptotics. The reason for this often is that regularity of $\mu$ permits localization, allowing one to show that one can dispense with the behavior of polynomials outside a given neighborhood of a point (in an appropriate sense and setting of course). This is achieved by using polynomials that decay geometrically away from a given points, together with some version of (1.2).

This is particularly the case in studying asymptotics of Christoffel functions

$$
\lambda_{n}(d \mu, x)=\inf _{\operatorname{deg}(P) \leq n-1} \frac{\int P^{2} d \mu}{P^{2}(x)},
$$

where the inf is taken over all polynomials $P$ of degree $\leq n-1$. As is well known,

$$
\lambda_{n}(d \mu, x)=1 / \sum_{j=0}^{n-1} p_{j}^{2}(x)=1 / K_{n}(x, x),
$$

where

$$
K_{n}(x, t)=\sum_{j=0}^{n-1} p_{j}(x) p_{j}(t)
$$

is the $n$th reproducing kernel.

Vili Totik [31] established the following result, which is the single most important asymptotic for Christoffel functions. In its formulation, we need the notion of a regular set (not to be confused with a regular measure!). We say $E$ is a regular set if $\mathbb{C} \backslash E$ is a regular domain with respect to the Dirichlet problem. In particular any finite union of intervals is regular.

\section{Theorem 1.1}

Let $\mu$ be a measure with compact support $E$. Assume that $E$ is a regular set, and that $\mu$ is regular in the sense of Stahl, Totik and Ullmann. If $I$ is an interval in the support for which

$$
\int_{I} \log \mu^{\prime}>-\infty
$$


then for a.e. $x \in I$,

$$
\lim _{n \rightarrow \infty} n \lambda_{n}(d \mu, x)=\mu^{\prime}(x) / \nu_{E}^{\prime}(x) .
$$

Here $\nu_{E}^{\prime}(x)$ is the density of the equilibrium measure $\nu_{E}$ for $E$. Recall that if $E$ is a compact set in the plane, with positive logarithmic capacity, it has an equilibrium measure $\nu_{E}$. This is a probability measure such that the equilibrium potential

$$
V^{\nu_{E}}(z)=\int \log \frac{1}{|t-z|} d \nu_{E}(t)
$$

satisfies

$$
V^{\nu}(z)=-\log \operatorname{cap}(E)
$$

quasi-everywhere on $E$. Moreover, this equation holds precisely at every point of $E$ that is regular for the Dirichlet problem for $\mathbb{C} \backslash E$ - the so-called regular points. For further orientation, see [22], [23], [29].

In the special case $E=[-1,1], \nu_{E}^{\prime}(x)=\frac{1}{\pi \sqrt{1-x^{2}}}$, and Theorem 1.1 was established earlier by Maté, Nevai and Totik [18]. Totik used regularity in localization, which permitted replacing difficult measures $\mu$ by locally "nicer" ones. Totik observes in [31] that some sort of global condition like regularity is necessary. He notes that, given any compact set $E$, properly containing an interval $I$, one can construct a non-regular measure that satisfies the local Szegő condition (1.3), but for which (1.4) fails at every point of $I$. However, this still leaves open the question as to what global condition is necessary, and whether the Szegö condition (1.3) is necessary.

Recently, Barry Simon [27] proved that if $\mu$ is regular, with compact support $E$, and $\mu^{\prime}>0$ a.e. on an interval $I$, then

$$
\lim _{n \rightarrow \infty} \int_{I}\left|\nu_{E}^{\prime}-\frac{\mu^{\prime}}{n \lambda_{n}}\right|=0
$$

An essentially weaker result than asymptotics for Christoffel functions are ratio asymptotics, for example involving two closely related measures. This study goes back to a celebrated memoir of P. Nevai [20]. Typically, one might consider a non-negative function $g$ that is integrable with respect to $d \mu$, and try show that

$$
\lim _{n \rightarrow \infty} \frac{\lambda_{n}(g d \mu, x)}{\lambda_{n}(d \mu, x)}=g(x),
$$

in some sense. Note that this type of limit offers the hope of great generality, as its formulation does not involve equilibrium measures, or properties of the support. In particular, when $\mu$ is regular, and $g^{ \pm 1}$ are bounded on $\operatorname{supp}[\mu]$, while $g$ is continuous at $x$, then methods pioneered by P. Nevai allow one to establish (1.5). This subject was further explored by Mate, Nevai and Totik for orthogonal polynomials on the unit circle [17], and by Lopez [11] for measures on the whole real line

A recent result of the author [15] shows that, at least for ratio asymptotics of Christoffel functions, it is possible to move beyond the class of regular measures. In fact, (1.5) holds in measure for arbitrary compactly supported measures:

\section{Theorem 1.2}

Let $\mu$ be a compactly supported measure on the real line with infinitely many points 
in its support. Let $g: \mathbb{R} \rightarrow(0, \infty)$ be a d $\mu$ measurable function such that $g^{ \pm 1}$ are bounded on $\operatorname{supp}[\mu]$. Let $\varepsilon>0$. Then, as $n \rightarrow \infty$,

$$
\text { meas }\left\{x \in\left\{\mu^{\prime}>0\right\}:\left|\frac{\lambda_{n}(g d \mu, x)}{\lambda_{n}(d \mu, x)}-g(x)\right|>\varepsilon\right\} \rightarrow 0 .
$$

Moreover, for every $p>0$,

$$
\lim _{n \rightarrow \infty} \int_{\left\{\mu^{\prime}>0\right\}}\left|\frac{\lambda_{n}(g d \mu, x)}{\lambda_{n}(d \mu, x)}-g(x)\right|^{p} d x=0 .
$$

Here, of course, $\left\{\mu^{\prime}>0\right\}=\left\{x: \mu^{\prime}(x)>0\right\}$ and meas denotes linear Lebesgue measure. The essential feature of this result is the absence of local and global restrictions on $\mu$.

One important application of Totik's Theorem 1.1 is to universality limits for random matrices in the bulk of the spectrum. This much studied limit takes the form

$$
\lim _{n \rightarrow \infty} \frac{\tilde{K}_{n}\left(\xi+\frac{a}{\tilde{K}_{n}(\xi, \xi)}, \xi+\frac{b}{\tilde{K}_{n}(\xi, \xi)}\right)}{\tilde{K}_{n}(\xi, \xi)}=\frac{\sin \pi(a-b)}{\pi(a-b)},
$$

uniformly for $a, b$ in compact subsets of the real line. Here $\xi$ lies in the interior of $\operatorname{supp}[\mu]$, and

$$
\tilde{K}_{n}(s, t)=\mu^{\prime}(s)^{1 / 2} \mu^{\prime}(t)^{1 / 2} K_{n}(s, t)
$$

is a normalized form of the reproducing kernel. Quite often, we remove the normalization from the outer $K_{n}$, so that (1.8) takes the form

$$
\lim _{n \rightarrow \infty} \frac{K_{n}\left(\xi+\frac{a}{\tilde{K}_{n}(\xi, \xi)}, \xi+\frac{b}{\tilde{K}_{n}(\xi, \xi)}\right)}{K_{n}(\xi, \xi)}=\frac{\sin \pi(a-b)}{\pi(a-b)},
$$

with $a, b$ now lying in compact subsets of the complex plane.

The limits (1.8) and (1.9) arise in describing the correlation of spacings of eigenvalues of $n \times n$ Hermitian matrices with random entries. A probability distribution is placed on the space of such matrices, with a probability density that is related to the measure $\mu$ above. There are many settings for universality limits. In the most important cases, the fixed measure $\mu$ is replaced by measures that change with $n$. See [2], [3], [5], [6], [7],[9], [10], [19], [28], [30] for further orientation.

One of the biggest challenges is to determine the minimal conditions on $\mu$ that permit the universality limit (1.8). This has been intensively investigated in recent years, with important advances in [1], [8], [12], [13], [14], [25], [32]. To date, the most general result for fixed measures is due to Totik, and uses Theorem 1.1 above, as well as its method of proof:

\section{Theorem 1.3}

Let $\mu$ be a measure with compact support. Assume that $\mu$ is regular. If $I$ is an interval in the support for which

$$
\int_{I} \log \mu^{\prime}>-\infty
$$

then for a.e. $\xi \in I,(1.8)$ holds uniformly for $a, b$ in compact subsets of the real line.

Totik established this theorem using asymptotics for Christoffel functions, an inequality of the author, and the method of polynomial pullbacks. That allows one 
to pass from supp $[\mu]$ consisting of a single interval to several intervals, and then to general compact sets. Simon proved related results using Jost functions [25].

The drawback of this theorem is the global assumption of regularity, even though this is a weak global assumption. The author [12] came up with an alternative method to establish (1.9) that avoids the assumption of regularity. Its basic hypothesis is that (1.9) holds for $b=a$, that is

$$
\lim _{n \rightarrow \infty} \frac{K_{n}\left(\xi+\frac{a}{\tilde{K}_{n}(\xi, \xi)}, \xi+\frac{a}{\tilde{K}_{n}(\xi, \xi)}\right)}{K_{n}(\xi, \xi)}=1,
$$

for all real $a$, together with some local hypothesis, such as $\mu^{\prime}$ bounded above and below in some interval. Note that (1.10) can be reformulated as a ratio asymptotic for Christoffel functions,

$$
\lim _{n \rightarrow \infty} \frac{\lambda_{n}(d \mu, \xi)}{\lambda_{n}\left(d \mu, \xi+\frac{a}{\tilde{K}_{n}(\xi, \xi)}\right)}=1 .
$$

This ought to be easier to establish than (1.8), because $\lambda_{n}(d \mu, x)$ (or $K_{n}(x, x)$ along the "diagonal") admits an extremal property. Unfortunately, there do not seem to be any techniques that establish (1.11) without first establishing the much stronger limit (1.4) in Totik's Theorem 1.1.

Recently, the author [16] has established that for arbitrary measures with compact support, universality holds in measure:

\section{Theorem 1.4}

Let $\mu$ be a measure with compact support and with infinitely many points in the support. Let $\varepsilon>0$ and $r>0$. Then as $n \rightarrow \infty$,

$$
\begin{aligned}
& \quad \text { meas }\left\{\xi \in\left\{\mu^{\prime}>0\right\}: \sup _{|u|,|v| \leq r}\left|\frac{K_{n}\left(\xi+\frac{u}{\tilde{K}_{n}(\xi, \xi)}, \xi+\frac{u}{K_{n}(\xi, \xi)}\right)}{K_{n}(\xi, \xi)}-\frac{\sin \pi(u-v)}{\pi(u-v)}\right| \geq \varepsilon\right\} \\
& \rightarrow \quad 0 \text { as } n \rightarrow \infty .
\end{aligned}
$$

Using the standard equivalence between convergence in measure, and subsequences that converge a.e., one deduces:

\section{Corollary 1.5}

Assume the hypotheses of Theorem 1.4. Let $\mathcal{S}$ be an infinite sequence of positive integers. Then there is a subsequence $\mathcal{S}^{\prime}$ of $\mathcal{S}$ such that for a.e. $\xi \in\left\{\mu^{\prime}>0\right\}$,

$$
\lim _{n \rightarrow \infty, n \in \mathcal{S}^{\prime}} \frac{K_{n}\left(\xi+\frac{a}{\tilde{K}_{n}(\xi, \xi)}, \xi+\frac{b}{\tilde{K}_{n}(\xi, \xi)}\right)}{K_{n}(\xi, \xi)}=\frac{\sin \pi(a-b)}{\pi(a-b)},
$$

uniformly for $a, b$ in compact subsets of the plane.

The proof of Theorem 1.4 is complicated. It depends on a uniqueness theorem for the sinc kernel, on maximal functions, and Hilbert transforms, and the theory of entire functions of exponential type.

It is no coincidence that convergence in measure is the conclusion in Theorems 1.2 and 1.4. Both depend heavily on upper bounds for the reproducing kernel $K_{n}$ that are true outside sets of small measure. The latter depend on bounds on Green's 
functions associated with $\mathbb{C} \backslash E$, where $E$ is an arbitrary compact subset of the real line.

Another key tool in both Theorems 1.2 and 1.4 is an estimate for the tail integral

$$
\Psi_{n}(x, r)=\frac{\int_{|t-x| \geq \frac{r}{K_{n}(x, x)}} K_{n}(x, t)^{2} d \mu(t)}{K_{n}(x, x)}, r>0 .
$$

Here if $\mu^{\prime}(x)=0$, or does not exist, we set $\Psi_{n}(x, r)=0$. Also, let

$$
A_{n}(x)=p_{n-1}^{2}(x)+p_{n}^{2}(x)
$$

and define the maximal function

$$
\mathcal{M}[d \nu](x)=\sup _{h>0} \frac{1}{2 h} \int_{x-h}^{x+h} d \nu
$$

for positive measures $\nu$ on the real line. In [15], we showed that for a.e. $x \in \operatorname{supp}[\mu]$,

$$
\Psi_{n}(x, r) \leq \frac{8}{r}\left(\frac{\gamma_{n-1}}{\gamma_{n}} \mathcal{M}\left[A_{n} d \mu\right](x)\right)^{2} .
$$

Using the classical weak $(1,1)$ estimate for maximal functions readily yields, for $r, \varepsilon>0$,

$$
\text { meas }\left\{x \in \operatorname{supp}[\mu]: \Psi_{n}(x, r) \geq \varepsilon\right\} \leq \frac{\gamma_{n-1}}{\gamma_{n}} \frac{17}{\sqrt{r \varepsilon}} .
$$

This estimate has some applications to what Barry Simon calls the Nevai condition. One way to formulate this involves the Nevai operators $\left\{G_{n}\right\}$. Given a function $f$ that is integrable with respect to $d \mu$, we define

$$
G_{n}[d \mu, f](x)=\frac{\int K_{n}^{2}(x, t) f(t) d \mu(t)}{K_{n}(x, x)} .
$$

The Nevai condition at $x$ is that

$$
\lim _{n \rightarrow \infty} G_{n}[d \mu, f](x)=f(x)
$$

for every continuous $f$. Paul Nevai [20] introduced the operators $\left\{G_{n}\right\}$ as a means to establishing the ratio asymptotic (1.5) for Christoffel functions.

A very interesting recent result of Breuer, Last, and Simon [4], relates the Nevai condition to sub-exponential growth of orthogonal polynomials:

\section{Theorem 1.6}

Assume that

$$
0<\inf _{n} \frac{\gamma_{n-1}}{\gamma_{n}} \leq \sup _{n} \frac{\gamma_{n-1}}{\gamma_{n}}<\infty .
$$

Then (1.17) holds at $x$ for every continuous compactly supportly function $f$ iff

$$
\lim _{n \rightarrow \infty} \frac{p_{n}^{2}(x)}{\sum_{j=0}^{n} p_{j}^{2}(x)}=0 .
$$

An equivalent formulation of (1.19) is that

$$
\lim _{n \rightarrow \infty} \frac{\lambda_{n-1}(d \mu, x)}{\lambda_{n}(d \mu, x)}=1 .
$$

Sub-exponential growth of orthogonal polynomials has been studied intensively over the years [20], [21]. It was Nevai and his collaborators who showed that when the 
measure $\mu$ has $[-1,1]$ as its essential support, and its recurrence coefficients have appropriate limits, then (1.19) is true throughout $[-1,1]$. In particular, this is true when $\mu^{\prime}>0$ a.e. in $[-1,1]$. More recently, Breuer, Last and Simon [4] constructed an example of a regular measure with support $[-2,2]$ such that (1.19) fails at every point of (for example) [1,2]. Nevertheless, they formulated the following:

\section{Conjecture 1.7}

Let $\mu$ have compact support. The Nevai condition (1.19) holds for d $\mu$ a.e. $x \in \operatorname{supp}[d \mu]$.

Here we shall prove the following simple:

\section{Theorem 1.8}

Let $\mu$ be compactly supported with infinitely many points in its support. Let $\left\{n_{k}\right\}_{k=1}^{\infty}$ be an increasing sequence of positive integers with

$$
\sum_{k=1}^{\infty} \frac{1}{n_{k}}<\infty
$$

(a) Then for Lebesgue a.e. $x \in\left\{\mu^{\prime}>0\right\}$,

$$
\lim _{k \rightarrow \infty} A_{n_{k}}(x) / K_{n_{k}}(x, x)=0 .
$$

(b) Let $f: \mathbb{R} \rightarrow \mathbb{R}$ be continuous and of compact support. Then for Lebesgue a.e. $x \in\left\{\mu^{\prime}>0\right\}$,

$$
\lim _{k \rightarrow \infty} G_{n_{k}}[f](x)=f(x)
$$

(c) Let $g: \mathbb{R} \rightarrow \mathbb{R}$ be continuous, of compact support, and positive on supp $[\mu]$. Then for Lebesgue a.e. $x \in\left\{\mu^{\prime}>0\right\}$,

$$
\lim _{k \rightarrow \infty} \frac{\lambda_{n_{k}}(g d \mu, x)}{\lambda_{n_{k}}(d \mu, x)}=g(x) .
$$

Recall that $A_{n}$ was defined at (1.15). Since a sequence of functions converges in measure iff every subsequence contains another subsequence that converges a.e., Theorem 1.8(c) has the following consequence: as $n \rightarrow \infty, \frac{\lambda_{n}(g d \mu, \cdot)}{\lambda_{n}(d \mu, \cdot)} \rightarrow g$ in measure in $\left\{\mu^{\prime}>0\right\}$. This provides an alternative, and simpler, proof of the special case of Theorem 1.2 in which $g$ is continuous.

We shall discuss the application of (1.16) to estimates of $A_{n}(x) / K_{n}(x, x)$, and prove Theorem 1.8 in the next section.

\section{Proof of Theorem 1.8}

We may assume that $\operatorname{supp}[\mu]$ is contained in $[-1,1]$. Maté, Nevai, and Totik $[18]$ proved, without any further restrictions on $\mu$, that

$$
\begin{aligned}
\limsup _{n \rightarrow \infty} n \lambda_{n}(d \mu, x) & \leq \mu^{\prime}(x) / \nu_{[-1,1]}(x) \\
& =\pi \sqrt{1-x^{2}} \mu^{\prime}(x)
\end{aligned}
$$

for a.e. $x \in \operatorname{supp}[\mu]$. It follows that if we let

$$
\mathcal{S}_{j}=\left\{\mu^{\prime}>0\right\} \cap\left\{x: n \lambda_{n}(d \mu, x) \leq 4 \mu^{\prime}(x) \text { for all } n \geq j\right\},
$$


then

$$
\mathcal{G}=\operatorname{supp}[\mu] \backslash \bigcup_{j=1}^{\infty} \mathcal{S}_{j} \text { has meas }(\mathcal{G})=0 .
$$

Note that

$$
x \in \mathcal{S}_{j} \Rightarrow \frac{n}{\tilde{K}_{n}(x, x)} \leq 4 \text { for all } n \geq j .
$$

We recall that $\Psi_{n}(x, r)$ was defined by (1.14). We also let

$$
\Omega_{n}(x, r)=\frac{\int_{|t-x| \geq r} K_{n}^{2}(x, t) d \mu(t)}{K_{n}(x, x)},
$$

and $d$ denotes the diameter of $\operatorname{supp}[\mu]$.

Our first estimate is a consequence of (1.16):

\section{Lemma 2.1}

Let $n \geq j \geq 1$, and $\varepsilon, r>0$.

(a)

$$
\operatorname{meas}\left(\mathcal{S}_{j} \cap\left\{x: \Omega_{n}(x, r) \geq \varepsilon\right\}\right) \leq \frac{\gamma_{n-1}}{\gamma_{n}} \frac{34}{\sqrt{n r \varepsilon}} \text {. }
$$

(b)

$$
\begin{aligned}
& \operatorname{meas}\left(\mathcal{S}_{j} \cap\left\{x: \frac{A_{n}(x)}{K_{n}(x, x)} \geq \varepsilon\right\}\right) \\
\leq & \left(\frac{\gamma_{n-1}}{\gamma_{n}}\right)^{-1 / 2} \frac{60 d}{\sqrt{n} \varepsilon^{3 / 4}} .
\end{aligned}
$$

Proof

(a) For $x \in \mathcal{S}_{j}$ and $n \geq j$, we have by (2.2),

$$
\Omega_{n}(x, r) \leq \frac{\int_{|t-x| \geq r} \frac{n}{4 \bar{K}_{n}(x, x)} K_{n}^{2}(x, t) d \mu(t)}{K_{n}(x, x)}=\Psi_{n}\left(x, \frac{n r}{4}\right) .
$$

Thus

$$
\begin{aligned}
& \operatorname{meas}\left(\mathcal{S}_{j} \cap\left\{x: \Omega_{n}(x, r) \geq \varepsilon\right\}\right) \\
\leq & \operatorname{meas}\left(\mathcal{S}_{j} \cap\left\{x: \Psi_{n}\left(x, \frac{n r}{4}\right) \geq \varepsilon\right\}\right) \\
\leq & \frac{\gamma_{n-1}}{\gamma_{n}} \frac{17(2)}{\sqrt{n r \varepsilon}}
\end{aligned}
$$

by (1.16).

(b) We use an idea of Breuer, Last and Simon [4]: from the Christoffel-Darboux formula, and orthogonality,

$$
\int(t-x)^{2} K_{n}^{2}(x, t) d \mu(t)=\left(\frac{\gamma_{n-1}}{\gamma_{n}}\right)^{2} A_{n}(x) .
$$


Then, given $\eta>0$, we see that

$$
\begin{aligned}
& \left(\frac{\gamma_{n-1}}{\gamma_{n}}\right)^{2} \frac{A_{n}(x)}{K_{n}(x, x)} \\
\leq & \eta^{2} \frac{\int_{|t-x| \leq \eta} K_{n}^{2}(x, t) d \mu(t)}{K_{n}(x, x)}+d^{2} \frac{\int_{|t-x|>\eta} K_{n}^{2}(x, t) d \mu(t)}{K_{n}(x, x)} \\
\leq & \eta^{2}+d^{2} \Omega_{n}(x, \eta) .
\end{aligned}
$$

Then

$$
\left(\frac{\gamma_{n-1}}{\gamma_{n}}\right)^{2} \frac{A_{n}(x)}{K_{n}(x, x)} \geq 2 \eta^{2} \Rightarrow d^{2} \Omega_{n}(x, \eta) \geq \eta^{2}
$$

so

$$
\begin{aligned}
& \text { meas }\left(\mathcal{S}_{j} \cap\left\{x: \frac{A_{n}(x)}{K_{n}(x, x)} \geq\left(\frac{\gamma_{n-1}}{\gamma_{n}}\right)^{-2} 2 \eta^{2}\right\}\right) \\
\leq & \text { meas }\left(\mathcal{S}_{j} \cap\left\{x: \Omega_{n}(x, \eta) \geq \frac{\eta^{2}}{d^{2}}\right\}\right) \leq \frac{\gamma_{n-1}}{\gamma_{n}} \frac{34 d}{\sqrt{n} \eta^{3 / 2}},
\end{aligned}
$$

by (a). Now make the substitution

$$
\varepsilon=\left(\frac{\gamma_{n-1}}{\gamma_{n}}\right)^{-2} 2 \eta^{2}
$$

to obtain (2.5).

We can obtain an alternative estimate, by more elementary means. It has a larger, better power of $n$ in the denominator, but also a worse power of $\varepsilon$ :

\section{Lemma 2.2}

Let $n \geq j \geq 1$, and $\varepsilon, r>0$. We have

(a)

$$
\begin{aligned}
& \text { meas }\left(\mathcal{S}_{j} \cap\left\{x: \frac{A_{n}(x)}{K_{n}(x, x)} \geq \varepsilon\right\}\right) \\
\leq & \frac{8}{n \varepsilon} .
\end{aligned}
$$

(b)

$$
\begin{aligned}
& \operatorname{meas}\left(\mathcal{S}_{j} \cap\left\{x: \Omega_{n}(x, r) \geq \varepsilon\right\}\right) \\
\leq & \frac{8}{n \varepsilon}\left(\frac{1}{r} \frac{\gamma_{n-1}}{\gamma_{n}}\right)^{2} .
\end{aligned}
$$

\section{Proof}

(a) For $x \in \mathcal{S}_{j},(2.2)$ shows that

$$
\frac{A_{n}(x)}{K_{n}(x, x)}=\frac{A_{n}(x) \mu^{\prime}(x)}{n} \frac{n}{\tilde{K}_{n}(x, x)} \leq \frac{4 A_{n}(x) \mu^{\prime}(x)}{n},
$$


so,

$$
\begin{aligned}
& \operatorname{meas}\left(\mathcal{S}_{j} \cap\left\{x: \frac{A_{n}(x)}{K_{n}(x, x)} \geq \varepsilon\right\}\right) \\
\leq & \operatorname{meas}\left(\mathcal{S}_{j} \cap\left\{x: A_{n}(x) \mu^{\prime}(x) \geq \frac{n \varepsilon}{4}\right\}\right) \\
\leq & \frac{4}{n \varepsilon} \int A_{n}(x) \mu^{\prime}(x) d x \leq \frac{8}{n \varepsilon} .
\end{aligned}
$$

(b) From (2.6),

$$
\begin{aligned}
& \int_{|t-x| \geq r} K_{n}^{2}(x, t) d \mu(t) \\
\leq & \int\left(\frac{t-x}{r}\right)^{2} K_{n}^{2}(x, t) d \mu(t)=\left(\frac{\gamma_{n-1}}{r \gamma_{n}}\right)^{2} A_{n}(x),
\end{aligned}
$$

so

$$
\Omega_{n}(x, r) \leq\left(\frac{\gamma_{n-1}}{r \gamma_{n}}\right)^{2} \frac{A_{n}(x)}{K_{n}(x, x)}
$$

Thus,

$$
\begin{aligned}
& \text { meas }\left(\mathcal{S}_{j} \cap\left\{x: \Omega_{n}(x, r) \geq \varepsilon\right\}\right) \\
\leq & \text { meas }\left(\mathcal{S}_{j} \cap\left\{x: \frac{A_{n}(x)}{K_{n}(x, x)} \geq \varepsilon\left(\frac{\gamma_{n-1}}{r \gamma_{n}}\right)^{-2}\right\}\right) \\
\leq & \frac{8}{n \varepsilon}\left(\frac{\gamma_{n-1}}{r \gamma_{n}}\right)^{2} .
\end{aligned}
$$

We turn to the

\section{Proof of Theorem 1.8}

(a) Fix $j \geq 1$, and let

$$
\mathcal{E}_{n}(j, \varepsilon)=\mathcal{S}_{j} \cap\left\{x: \frac{A_{n}(x)}{K_{n}(x, x)} \geq \varepsilon\right\} .
$$

For $n \geq j$, Lemma 2.2(a) gives

$$
\operatorname{meas}\left(\mathcal{E}_{n}(j, \varepsilon)\right) \leq \frac{8}{n \varepsilon} .
$$

Let

$$
\mathcal{E}(j, \varepsilon)=\limsup _{k \rightarrow \infty} \mathcal{E}_{n_{k}}(j, \varepsilon)=\bigcap_{\ell=1}^{\infty} \bigcup_{k=\ell}^{\infty} \mathcal{E}_{n_{k}}(j, \varepsilon) .
$$

Because of $(1.20), \mathcal{E}(j, \varepsilon)$ has linear Lebesgue measure 0. For $x \in\left\{\mu^{\prime}>0\right\} \backslash(\mathcal{E}(j, \varepsilon) \cup \mathcal{G})$, we have for large enough $k$,

$$
\frac{A_{n_{k}}(x)}{K_{n_{k}}(x, x)}<\varepsilon
$$

Recall that $\mathcal{G}$ was defined at (2.1). Then, if

$$
\mathcal{E}=\mathcal{G} \cup \bigcup_{j, \ell \geq 1} \mathcal{E}\left(j, \frac{1}{\ell}\right),
$$


we see that $\mathcal{E}$ has linear Lebesgue measure 0 , and for $x \in\left\{\mu^{\prime}>0\right\} \backslash \mathcal{E}$,

$$
\lim _{k \rightarrow \infty} \frac{A_{n_{k}}(x)}{K_{n_{k}}(x, x)}=0 .
$$

(b) Let

$$
\mathcal{F}_{n}(j, r, \varepsilon)=\mathcal{S}_{j} \cap\left\{x: \Omega_{n}(x, r) \geq \varepsilon\right\},
$$

so that by Lemma $2.2(\mathrm{~b})$,

$$
\operatorname{meas}\left(\mathcal{F}_{n}(j, r, \varepsilon)\right) \leq \frac{8}{n \varepsilon}\left(\frac{\gamma_{n-1}}{r \gamma_{n}}\right)^{2} \leq \frac{8}{n \varepsilon}\left(\frac{d}{r}\right)^{2} .
$$

Recall that $d$ is the diameter of $\operatorname{supp}[\mu]$. Let

$$
\mathcal{F}(j, r, \varepsilon)=\limsup _{k \rightarrow \infty} \mathcal{F}_{n_{k}}(j, r, \varepsilon),
$$

so that $\mathcal{F}(j, r, \varepsilon)$ has Lebesgue measure 0 , by (1.20) again. For $x \in\left\{\mu^{\prime}>0\right\} \backslash(\mathcal{G} \cup \mathcal{F}(j, r, \varepsilon))$, we have

Finally, let

$$
\Omega_{n_{k}}(x, r) \leq \varepsilon \text { for } k \text { large enough. }
$$

$$
\mathcal{F}=\mathcal{G} \cup \bigcup_{j, \ell, m \geq 1} \mathcal{F}\left(j, \frac{1}{\ell}, \frac{1}{m}\right) .
$$

Then $\mathcal{F}$ has Lebesgue measure 0 , and for $x \in\left\{\mu^{\prime}>0\right\} \backslash \mathcal{F}$, we have, for each $r>0$,

$$
\lim _{k \rightarrow \infty} \Omega_{n_{k}}(x, r)=0 .
$$

Now let $f$ be continuous and of compact support. We see that

$$
\begin{aligned}
& \left|G_{n}[d \mu, f](x)-f(x)\right| \\
\leq & \frac{1}{K_{n}(x, x)} \int_{|t-x| \leq r}|f(t)-f(x)| K_{n}^{2}(x, t) d \mu(t) \\
& +2\|f\|_{L_{\infty}(\mathbb{R})} \Omega_{n}(x, r) \\
\leq & \sup _{|t-x| \leq r}|f(t)-f(x)|+2\|f\|_{L_{\infty}(\mathbb{R})} \Omega_{n}(x, r) .
\end{aligned}
$$

It follows that for $x \in\left\{\mu^{\prime}>0\right\} \backslash \mathcal{F}$,

$$
\limsup _{k \rightarrow \infty}\left|G_{n_{k}}[d \mu, f](x)-f(x)\right| \leq \sup _{|t-x| \leq r}|f(t)-f(x)| .
$$

As $r>0$ is arbitrary, continuity of $f$ gives (1.22).

(c) We use the elementary inequality $[20$, p. 76$]$

$$
\frac{\lambda_{n}(g d \mu, x)}{\lambda_{n}(d \mu, x)} \leq G_{n}[d \mu, g](x) .
$$

Together with (b), this gives, for a.e. $x \in\left\{\mu^{\prime}>0\right\}$,

$$
\limsup _{k \rightarrow \infty} \frac{\lambda_{n_{k}}(g d \mu, x)}{\lambda_{n_{k}}(d \mu, x)} \leq g(x) .
$$

Replacing $d \mu$ by $g d \mu$, and $g$ by $g^{-1}$, gives for a.e. $x \in\left\{\mu^{\prime}>0\right\}$ (recall that $g$ is bounded below in compact supp $[\mu]$ ),

$$
\limsup _{k \rightarrow \infty} \frac{\lambda_{n_{k}}(d \mu, x)}{\lambda_{n_{k}}(g d \mu, x)} \leq g^{-1}(x) .
$$

Then (1.23) follows. 


\section{REFERENCES}

[1] A. Avila, Y. Last, and B. Simon, Bulk universality and clock spacing of zeros for ergodic Jacobi matrices with a.c. spectrum, submitted.

[2] J. Baik, T. Kriecherbauer, K. T-R. McLaughlin, P.D. Miller, Uniform Asymptotics for Polynomials Orthogonal with respect to a General Class of Discrete Weights and Universality Results for Associated Ensembles, Princeton Annals of Mathematics Studies, 2006.

[3] P. Bleher and A. Its, Random matrix models and their applications, Cambridge University Press, Cambridge, 2001.

[4] J. Breuer, Y. Last, B. Simon, The Nevai Condition, to appear in Constr. Approx.

[5] P. Deift, Orthogonal Polynomials and Random Matrices: A Riemann-Hilbert Approach, Courant Institute Lecture Notes, Vol. 3, New York University Pres, New York, 1999.

[6] P. Deift, D. Gioev, Random Matrix Theory: Invariant Ensembles and Universality, Courant Institute Lecture Notes, Vol. 18, New York University Pres, New York, 2009.

[7] P. Deift, T. Kriecherbauer, K. T-R. McLaughlin, S. Venakides and X. Zhou, Uniform Asymptotics for Polynomials Orthogonal with respect to Varying Exponential Weights and Applications to Universality Questions in Random Matrix Theory, Communications in Pure and Applied Maths., 52(1999), 1335-1425.

[8] E. Findley, Universality for Regular Measures satisfying Szegö's Condition, J. Approx. Theory, 155 (2008), 136-154.

[9] P. J. Forrester, Log-gases and Random matrices, online book, http://www.ms.unimelb.edu.au/ ${ }^{\sim}$ matpjf/matpjf.html

[10] Eli Levin and D.S. Lubinsky, Universality Limits in the Bulk for Varying Measures, Advances in Mathematics, 219(2008), 743-779.

[11] G. Lopez, Relative Asymptotics for Polynomials Orthogonal on the Real Axis, Math. USSR. Sbornik, 65(1990), 505-529.

[12] D.S. Lubinsky, Universality limits in the bulk for arbitrary measures on compact sets, J. d'Analyse Mathematique, 106 (2008), 373-394.

[13] D.S. Lubinsky, A New Approach to Universality Limits involving Orthogonal Polynomials, Annals of Mathematics, 170(2009), 915-939.

[14] D.S. Lubinsky, Universality Limits for Random Matrices and de Branges Spaces of Entire Functions, Journal of Functional Analysis, 256(2009), 3688-3729.

[15] D.S. Lubinsky, A Maximal Function Approach to Christoffel Functions and Nevai's Operators, to appear in Constructive Apprioximation.

[16] D.S. Lubinskt, Bulk Universality Holds in Measure for Compactly Supported Measures, manuscript..

[17] A. Maté, P. Nevai, V. Totik, Extensions of Szegö's Theory of Orthogonal Polynomials, II, Constr. Approx., 3(1987), 51-72.

[18] A. Mate, P. Nevai, V. Totik, Szeg”'s Extremum Problem on the Unit Circle, Annals of Mathematics, 134(1991), 433-453.

[19] M.L. Mehta, Random Matrices, 2nd edn., Academic Press, Boston, 1991.

[20] P. Nevai, Orthogonal Polynomials, Memoirs of the AMS no. 213 (1979).

[21] P. Nevai, V.Totik, and J. Zhang, Orthogonal Polynomials: their growth relative to their Sums, J. Approx. Theory, 67(1991), 215-234.

[22] T. Ransford, Potential Theory in the Complex Plane, Cambridge University Press, Cambridge, 1995.

[23] E.B. Saff and V. Totik, Logarithmic Potentials with External Fields, Springer, New York, 1997.

[24] B. Simon, Orthogonal Polynomials on the Unit Circle, Parts 1 and 2, American Mathematical Society, Providence, 2005.

[25] B. Simon, Two Extensions of Lubinsky's Universality Theorem, J. d'Analyse Mathematique, 105 (2008), 345-362.

[26] B. Simon, The Christoffel-Darboux Kernel, (in) Perspectives in PDE, Harmonic Analysis and Applications, a volume in honor of V.G. Maz'ya's 70th birthday, Proceedings of Symposia in Pure Mathematics, 79 (2008), 295-335.

[27] B. Simon, Weak Convergence of CD Kernels and Applications,

[28] A. Soshnikov, Universality at the Edge of the Spectrum in Wigner Random Matrices, Comm. Math. Phys., 207(1999), 697-733. 
[29] H. Stahl and V. Totik, General Orthogonal Polynomials, Cambridge University Press, Cambridge, 1992.

[30] T. Tao, V. Vu, From the Littlewood-Offord Problem to the Circular Law: Universality of the Spectral Distribution of Random Matrices, Bull. Amer. Math. Soc., 46 (2009), 377-396.

[31] V. Totik, Asymptotics for Christoffel Functions for General Measures on the Real Line, J. d' Analyse de Mathematique, 81(2000), 283-303.

[32] V. Totik, Universality and fine zero spacing on general sets, Arkiv för Matematik, 47(2009), 361-391.

School of Mathematics, Georgia Institute of Technology, Atlanta, Ga 30332-0160, USA., LUBINSKY@MATH.GATECH.EDU 